\title{
Levels and expressions of orotate phosphoribosyltransferase in gastric carcinoma and normal gastric mucosa tissues
}

\author{
Yoichi Sakurai $^{1}$, Shingo Kamoshida ${ }^{2}$, Shinpei Furuta ${ }^{1}$, Risaburo Sunagawa ${ }^{1}$, Kazuki Inaba ${ }^{1}$, Jun Isogaki ${ }^{1}$, \\ Yoshiyuki Komori ${ }^{1}$, Ichiro Uyama ${ }^{1}$, and Yutaka Tsutsumi ${ }^{2}$ \\ ${ }^{1}$ Department of Surgery, Fujita Health University School of Medicine, 1-98 Dengakugakubo Kutsukake-cho, Toyoake 470-1192, Japan \\ ${ }^{2}$ Department of Pathology, Fujita Health University School of Medicine, Toyoake, Japan
}

\begin{abstract}
Background. Orotate phosphoribosyltransferase (OPRT; EC 2.4.2.10), a key enzyme that catalyzes one of the primary steps in the phosphorylation of fluoropyrimidine, was recently recognized as an important enzyme that determines the anticancer effects of the dihydropyrimidine dehydrogenase-inhibitory fluoropyrimidine, S-1.

Methods. Levels of OPRT were examined in 97 gastric carcinoma tissues and 65 normal gastric mucosa tissues obtained from patients with gastric carcinoma. The relation between OPRT levels and clinicopathological variables was evaluated, and correlations of OPRT with thymidylate synthase and dihydropyrimidine dehydrogenase levels in gastric carcinoma tissues were evaluated.

Results. Although OPRT levels were high in welldifferentiated and localized carcinomas, they were not correlated with other clinicopathological variables or with the pathological stage of gastric carcinoma. Levels of OPRT were significantly higher in gastric carcinoma tissue than in normal gastric mucosa. OPRT levels were not correlated with levels of either thymidylate synthase or dihydropyrimidine dehydrogenase. In samples of gastric carcinoma tissues and normal gastric mucosa tissues obtained simultaneously from 24 patients, no correlation was found between OPRT levels in gastric carcinoma and levels in normal gastric mucosa.

Conclusion. These results suggest that the OPRT level is significantly higher in gastric carcinoma tissue than in normal gastric mucosa and that the OPRT level in gastric carcinoma is a novel variable that is independent of the levels of other previously known enzymes related to 5-fluorouracil (FU) metabolism.
\end{abstract}

Key words Orotate phosphoribosyltransferase - ELISA · 5-Fluorouracil · Chemosensitivity · Gastric cancer

Offprint requests to: Y. Sakurai

Received: September 10, 2007 / Accepted: October 17, 2007

\section{Introduction}

Although surgical resection is recognized as the primary treatment for patients with advanced gastric carcinoma, overall survival rates in such patients are not yet satisfactory $[1,2]$. Neoadjuvant chemotherapy (NAC) or postoperative adjuvant chemotherapy with anticancer agents with a high efficacy rate has thus been an essential adjunct to improve the clinical outcome of patients undergoing surgical resection [3]. A dihydropyrimidine dehydrogenase (DPD)-inhibitory fluoropyrimidine (DIF) is widely recognized as the most effective first-line chemotherapeutic agent for NAC or postoperative adjuvant chemotherapy in gastric carcinoma [4-7]; however, bona fide factors predictive of sensitivity to this agent remain to be elucidated. S-1 (TS-1; Taiho Pharmaceutical, Tokyo, Japan) is a novel oral DIF developed by Shirasaka et al. [8,9]. It is designed to provide high and prolonged serum concentrations of 5fluorouracil (5-FU) with minimal toxicity. S-1 contains tegafur (a masked compound of 5-FU) and two types of enzyme inhibitors, 5-chloro-2, 4-dihydropyrimidine and potassium oxonate, in a molar ratio of $1: 0.4: 1$.

Orotate phosphoribosyltransferase (OPRT; EC 2.4.2.10) is the key enzyme involved in the phosphorylation of 5-fluorouridine-5'-monophosphate (FUMP), with 5-phosphoribosyl-1-phosphate as the cosubstrate [10]. We previously showed that sensitivity to a DIF such as UFT (Taiho Pharmaceutical, Tokyo, Japan) was correlated with OPRT levels in human cancer xenografts [11]; therefore, OPRT may be an important enzyme for determining the anticancer effects of DIFs and for predicting the postoperative survival of patients with gastric carcinoma. We recently established an enzyme-linked immunosorbent assay (ELISA) that uses antibodies against the OPRT molecule to measure tissue OPRT levels [11-13]. The assay allows us to process large numbers of tissue samples simultaneously, with minimal amounts of tissue [11]. Our pre- 
liminary immunohistochemical results have shown that OPRT is expressed in both gastric carcinoma and normal gastric mucosa tissues. OPRT levels have not been measured previously in normal gastric mucosa, however, and the relationship between OPRT levels and clinicopathological variables and levels of thymidylate synthase (TS) and DPD have not been previously investigated.

In the present study, we examined OPRT levels in gastric carcinoma tissue and in normal gastric mucosa and evaluated the relationship between OPRT levels and clinicopathological variables and the correlation between OPRT levels and TS and DPD in carcinoma tissue.

\section{Patients, materials, and methods}

\section{Patients and tissue specimens}

A total of 138 patients with advanced gastric carcinoma gave their written informed consent for the use of the specimens in the study. The study was approved by the Institutional Ethics Review Board for Human Investigation at Fujita Health University. A total of 97 gastric carcinoma tissue specimens and 65 normal gastric mucosa samples were obtained from 97 of the 138 patients with gastric carcinoma, who underwent surgical resection at the first Educational Hospital, Fujita Health University School of Medicine. Gastric carcinoma specimens and normal gastric mucosa were simultaneously obtained from 24 patients. All specimens were collected immediately after surgical resection and were immersed in liquid nitrogen and frozen at $-80^{\circ} \mathrm{C}$ until analyzed.

The patients with gastric carcinoma consisted of 32 women and 65 men whose mean age was $67.2 \pm 11.5$ years. The clinicopathological characteristics of the 97 patients with gastric carcinoma are summarized in Table 1. The clinicopathological factors are expressed in accordance with the Japanese Classification of Gastric Carcinoma [14].

\section{Enzyme-linked immunosorbent assay (ELISA) for measurement of tissue OPRT levels}

A recently established sandwich-type ELISA [11-13] was used to measure OPRT levels in gastric carcinoma tissue and normal gastric mucosa. We showed previously that a significant correlation existed between OPRT levels and OPRT enzymatic activity [11]; therefore, the OPRT level determined by the ELISA directly reflects enzymatic activity. The ELISA is described in detail elsewhere [11]. Briefly, the assay was performed with anti-OPRT-A and anti-OPRT-C antibodies as the catcher and the tracer, respectively. Fifty microliters of $5.0 \mathrm{mg} / \mathrm{ml}$ anti-OPRT-C antibody, dissolved in $0.1 \mathrm{M}$ carbonate buffer ( $\mathrm{pH}$ 9.6), was coated onto an enzyme immunoassay (EIA) plate (Nalge Nunc International; Rochester, NY, USA) at $4^{\circ} \mathrm{C}$ for $16 \mathrm{~h}$. After a washing with distilled water, blocking was performed with SabilCoat (SurModics; Eden Prairie, MN, USA) at room temperature for $60 \mathrm{~min}$. Protein extracts of cancer tissue samples were diluted and adjusted to a final concentration of $1 \mathrm{mg} / \mathrm{ml}$. One hundred milliliters of protein extract per well was added, and the plates were incubated at room temperature for $60 \mathrm{~min}$. The wells were then washed five times with phosphate-buffered saline (PBS). One hundred milliliters of peroxidase-labeled anti-OPRT-A antibody per well was added, and the plates were incubated at room temperature for $30 \mathrm{~min}$. After the wells were washed with PBS seven times, $100 \mathrm{ml}$ of orthophenylene diamine (OPD) dissolved in substrate buffer $\left(0.05 \mathrm{M}\right.$ citrate buffer, $0.01 \% \mathrm{H}_{2} \mathrm{O}_{2}$, $1 \mathrm{mM}$ ethylenediamine tetraacetic acid [EDTA]) solution was added, and the plates were incubated at room temperature for $30 \mathrm{~min}$. The reaction was stopped by the addition of $0.5 \mathrm{~N}$ sulfate solution. Absorbance at $492 \mathrm{~nm}$ was measured with a plate reader (Spectramax 3400PC; Molecular Devices, Tokyo, Japan). Protein extract derived from the human breast cancer cell line MDAMB-435S, in which OPRT protein was previously quantified by immunoblotting, was used as the standard. The OPRT level was determined by comparison with OPRT levels in the standard samples.

\section{Immunohistochemical expression of the OPRT molecule}

A total of 97 gastric carcinoma tissues were subjected to immunohistochemical staining with a polyclonal antibody against the human recombinant OPRT molecule. The production and characterization of the antibody are described in detail elsewhere [12]. Immunohistochemical staining was performed on formalin-fixed, paraffin-embedded sections of surgically resected specimens of gastric carcinoma, as described by Kamoshida et al. [15]. The sections were deparaffinized with xylene and rehydrated with graded ethanol solutions. Endogenous peroxidase was inactivated by $0.03 \%$ hydrogen peroxide in methanol for $30 \mathrm{~min}$. Rabbit polyclonal antibody against OPRT (diluted 1:500; Taiho Pharmaceutical, Tokushima, Japan) was added, and the sections were incubated overnight at room temperature. With the use of the universal immuno-enzyme polymer method, Histofine Simple Stain MAX-PO (Nichirei, Tokyo, Japan) was employed as a second-layer reagent. The reaction products were visualized in $50 \mathrm{mg} / \mathrm{dl} \mathrm{3,} \mathrm{3'-}$ diaminobenzidine tetrahydrochloride solution containing $0.003 \%$ hydrogen peroxide. The specificity of OPRT 
Table 1. OPRT levels in gastric carcinoma tissues, stratified by clinicopathological variables

\begin{tabular}{|c|c|c|}
\hline & $n$ & $\begin{array}{c}\text { OPRT level } \\
\text { (mean } \pm \text { SD) } \\
n=97\end{array}$ \\
\hline \multicolumn{3}{|l|}{ Histological type } \\
\hline Well-differentiated & 23 & $7.8 \pm 3.5$ \\
\hline Moderately differentiated & 36 & $5.2 \pm 3.0$ \\
\hline Poorly differentiated & 33 & $4.1 \pm 3.4^{*}$ \\
\hline Others & 5 & $4.8 \pm 4.4$ \\
\hline \multicolumn{3}{|l|}{ INF } \\
\hline$\alpha, \beta$ & 72 & $6.1 \pm 3.6$ \\
\hline$\gamma$ & 25 & $3.3 \pm 2.7 * *$ \\
\hline \multicolumn{3}{|l|}{$\mathrm{T}^{\prime}$} \\
\hline 1 & 6 & $6.7 \pm 2.2$ \\
\hline 2 & 18 & $5.6 \pm 3.7$ \\
\hline 3 & 67 & $5.2 \pm 3.6$ \\
\hline 4 & 6 & $5.7 \pm 4.4$ \\
\hline \multicolumn{3}{|l|}{ Stroma type } \\
\hline med & 6 & $5.9 \pm 4.3$ \\
\hline int & 76 & $5.7 \pm 3.6$ \\
\hline sci & 15 & $3.5 \pm 2.7 * * *$ \\
\hline \multicolumn{3}{|r|}{ - } \\
\hline 0 & 4 & $5.5 \pm 0.7$ \\
\hline 1 & 20 & $5.7 \pm 4.6$ \\
\hline 2 & 54 & $5.8 \pm 3.4$ \\
\hline 3 & 19 & $3.9 \pm 2.9$ \\
\hline \multicolumn{3}{|r|}{ 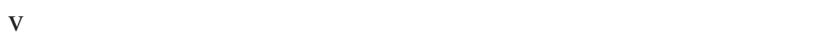 } \\
\hline 0 & 26 & $5.5 \pm 3.9$ \\
\hline 1 & 45 & $5.0 \pm 3.8$ \\
\hline 2 & 25 & $5.5 \pm 3.0$ \\
\hline 3 & 1 & $6.2 \pm 4.0$ \\
\hline \multicolumn{3}{|l|}{$\mathrm{n}$} \\
\hline 0 & 29 & $5.5 \pm 3.9$ \\
\hline 1 & 27 & $5.0 \pm 3.8$ \\
\hline 2 & 44 & $5.5 \pm 3.0$ \\
\hline 3 & 7 & $6.2 \pm 4.0$ \\
\hline \multicolumn{3}{|r|}{$0.2-1.0$} \\
\hline 0 & 87 & $5.5 \pm 3.6$ \\
\hline 1 & 10 & $4.3 \pm 3.0$ \\
\hline \multicolumn{3}{|l|}{$\mathrm{M}$} \\
\hline 0 & 92 & $5.3 \pm 3.6$ \\
\hline 1 & 5 & $7.8 \pm 2.7$ \\
\hline \multicolumn{3}{|l|}{ pStage } \\
\hline I & 12 & $6.5 \pm 3.7$ \\
\hline II & 18 & $4.9 \pm 4.0$ \\
\hline III & 50 & $5.0 \pm 3.1$ \\
\hline IV & 17 & $6.2 \pm 4.1$ \\
\hline
\end{tabular}

$* P<0.05$ vs well-differentiated adenocarcinoma; $* * P<0.05$ vs INF $\alpha, \beta ; * * *<0.05$ vs med type

staining was previously confirmed in preabsorption experiments [15]. Our previous study showed that OPRT immunoreactivity was observed in almost all tissue components at various intensities [15]. Therefore, the intensity of immunoreactivity in carcinoma tissue was compared with that of the surrounding normal gastric epithelium, and the immunostained sections were classified into two groups: positive (stronger than the normal epithelium) and negative (comparable with or weaker than the normal epithelium). All sections were reviewed independently by two pathologists blinded to all clinical and pathological information.

\section{Measurement of TS and DPD in human gastric carcinoma tissue}

Tissue concentrations of TS and DPD in 46 gastric carcinoma tissues were measured by using sandwich ELISAs established previously [16, 17]. The precise methods of each ELISA are described in detail elsewhere [17].

\section{Statistical analysis}

Differences in OPRT levels between gastric carcinoma tissue and normal gastric mucosa were evaluated by using Student's $t$-test and the Mann-Whitney test. Correlations between OPRT levels in carcinoma tissue and in normal gastric epithelium were evaluated by using correlation coefficients. The significance of the correlations was evaluated with Fischer's Z-transformation. All statistical calculations were performed by using Statview version 5.0 (Abacus Concepts, Berkeley, CA, USA). $P<0.05$ was considered statistically significant.

\section{Results}

OPRT levels in gastric carcinoma tissue and normal gastric mucosa

Levels of OPRT in gastric carcinoma tissue and normal gastric mucosa, stratified by clinicopathological variables, are summarized in Table 1. OPRT levels were significantly higher in well-differentiated type carcinomas than in poorly differentiated carcinomas and were higher in localized than in infiltrative types. OPRT levels were also significantly higher in cases with medullary type stroma than in cases with scirrhous type stroma. OPRT levels did not differ significantly according to T, ly, v, n, P, or M factors, or pathological stage. Distributions and histograms of OPRT levels in gastric carcinoma tissues and in normal gastric mucosa are shown in Fig. 1 A and Fig. 1 B, respectively. The mean OPRT level in gastric carcinoma tissues was $5.4 \pm 3.6 \mathrm{ng} /$ $\mathrm{mg}$ protein and that in normal gastric mucosa was $3.9 \pm$ $4.7 \mathrm{ng} / \mathrm{mg}$ protein $(P<0.05$ by Mann-Whitney test). The highest OPRT values in gastric carcinoma tissue and in normal gastric mucosa were $16.0 \mathrm{ng} / \mathrm{ml}$ and $19.9 \mathrm{ng} / \mathrm{ml}$, respectively. No correlation was found between the OPRT level in gastric carcinoma tissue and that in normal gastric mucosa obtained simultaneously from the same patients (Fig. 2; $r=0.218$; not significant [NS] by Fischer's Z-transformation). 

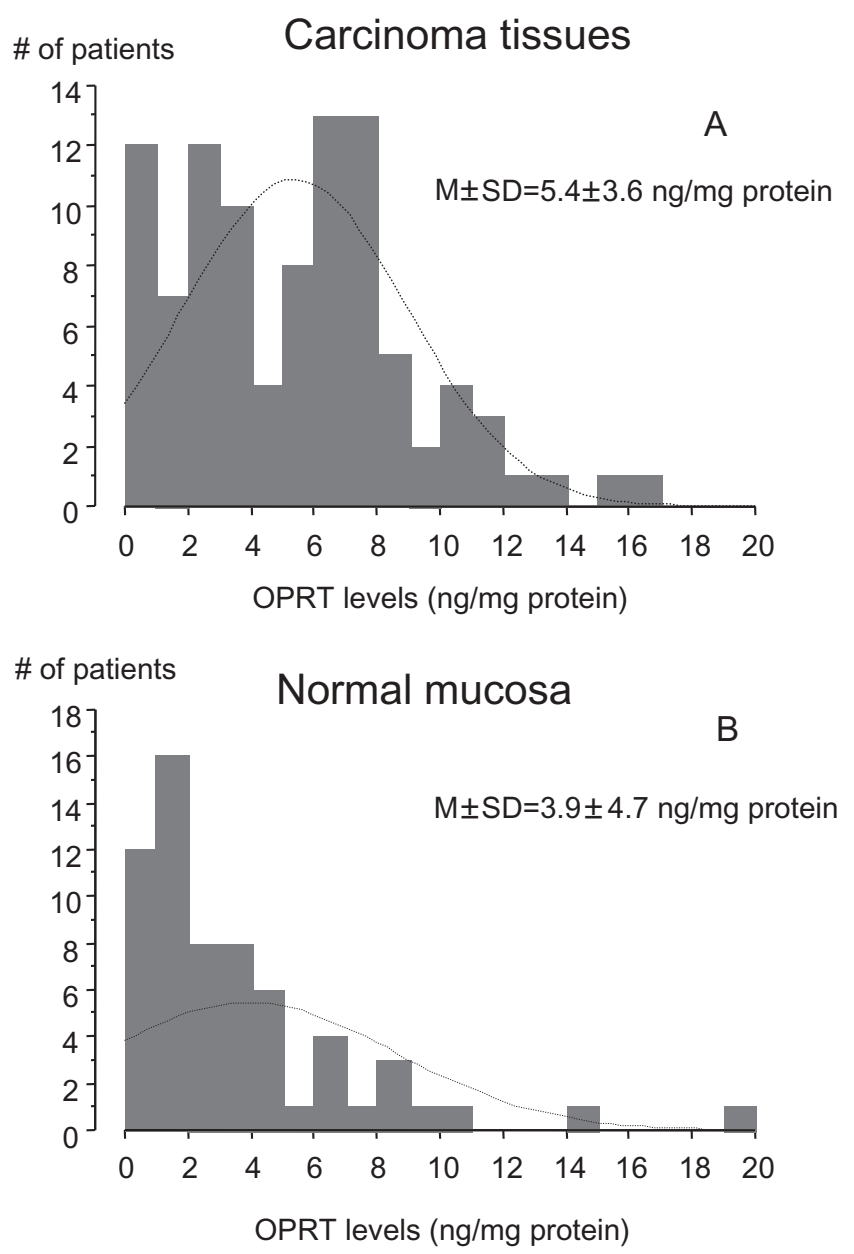

Fig. 1A,B. Distributions of orotate phosphoribosyltransferase $(O P R T)$ levels in gastric carcinoma tissue $(\mathbf{A})$ and normal gastric mucosa (B). $M$, mean

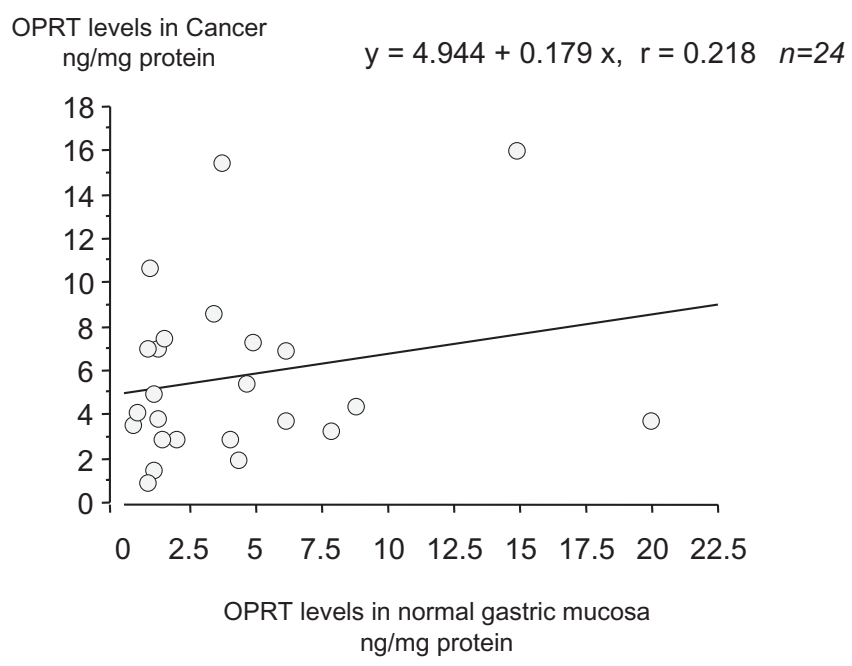

Fig. 2. Correlation between OPRT levels in gastric carcinoma tissues and in normal gastric mucosa $(r=0.218$, no significant correlation)

\section{Immunohistochemical reactivity to OPRT}

The immunohistochemical expression of OPRT in gastric carcinoma was examined in 97 cases. OPRT was immunohistochemically expressed not only in cancer cells but also in non-neoplastic gastric mucosa and in intestinal metaplastic cells (Fig. 3). Nonepithelial cells, such as neutrophils, lymphocytes, and smooth muscle cells, also stained positively. OPRT expression was positive in 25 of $97(25.8 \%)$ and negative in 72 of 97 (74.2\%) gastric carcinoma cases, respectively. The intensity of the immunohistochemical staining was stronger in intestinal-type gastric carcinoma than in diffuse-type gastric carcinoma (Fig. 3). The OPRT level (mean \pm SD) of the cases with positive OPRT expression was $7.5 \pm 2.5 \mathrm{ng} /$ $\mathrm{mg}$ protein and that of the cases with negative OPRT expression was $4.7 \pm 3.6 \mathrm{ng} / \mathrm{mg}$ protein $(P<0.0001$ by Mann-Whitney test).

\section{Correlation of OPRT level with TS and DPD levels in gastric carcinoma}

No significant correlations were found between OPRT levels and TS levels or between OPRT levels and DPD levels (Fig. 4 A, B). The correlation coefficients were -0.017 , and -0.354 , respectively (not significant by Fischer's Z-transformation).

\section{Discussion}

The primary aims of the present study were to examine OPRT levels in both gastric carcinoma tissue and normal gastric mucosa, to examine the correlation between OPRT levels and clinicopathological variables, and to examine the correlation between levels of OPRT and those of TS and DPD. Our results showed that OPRT levels were higher in patients with well-differentiated or localized-type carcinomas than in those with poorly differentiated or invasive types. There was no significant correlation between OPRT levels and clinicopathological variables. OPRT levels were significantly higher in gastric carcinoma tissue than in normal gastric mucosa. These results, along with our previous observations [11], suggest that OPRT plays an important role in the activation of fluoropyrimidines in carcinoma tissues and that the OPRT level in gastric carcinoma tissues is an independent variable that can predict sensitivity to dihydropyrimidine dehydrogenase-inhibitory fluoropyrimidines (DIFs).

Evidence for a significant anticancer effect of S-1 was shown by the recent interim analysis of adjuvant chemotherapy for gastric cancer with S-1 (ACTS-GC), randomized phase III multi-institutional clinical trial, as presented at the 2007 Gastrointestinal Cancers 


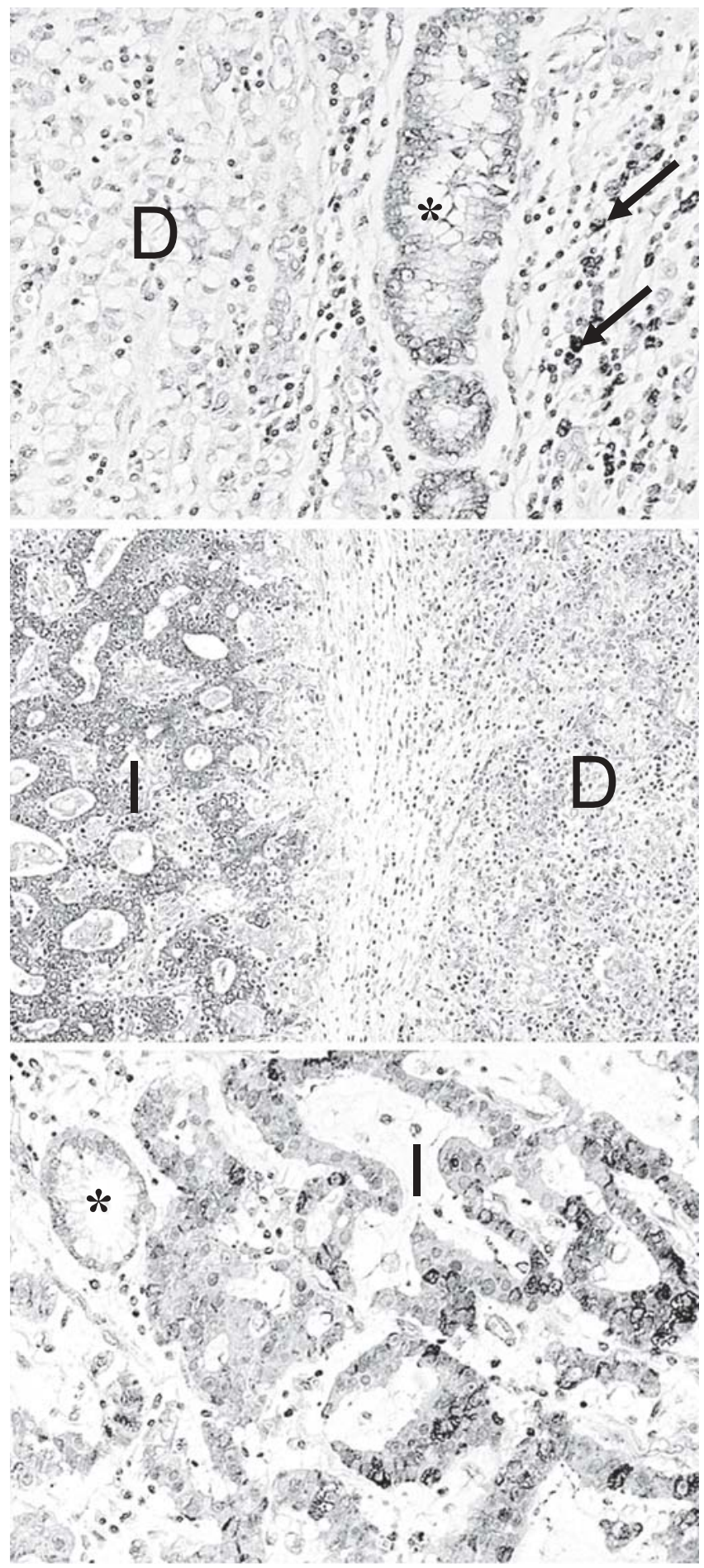

Fig. 3. OPRT immunoreactivity in gastric carcinoma tissue and normal gastric epithelium. Weak OPRT staining is noted in non-neoplastic gastric mucosa (asterisks). The intensity of OPRT immunoreactivity in intestinal-type gastric carcinoma $(I)$ is stronger than that in diffuse-type gastric carcinoma $(D)$ or normal epithelium. Neutrophils in the stroma (arrows) are also OPRT-positive
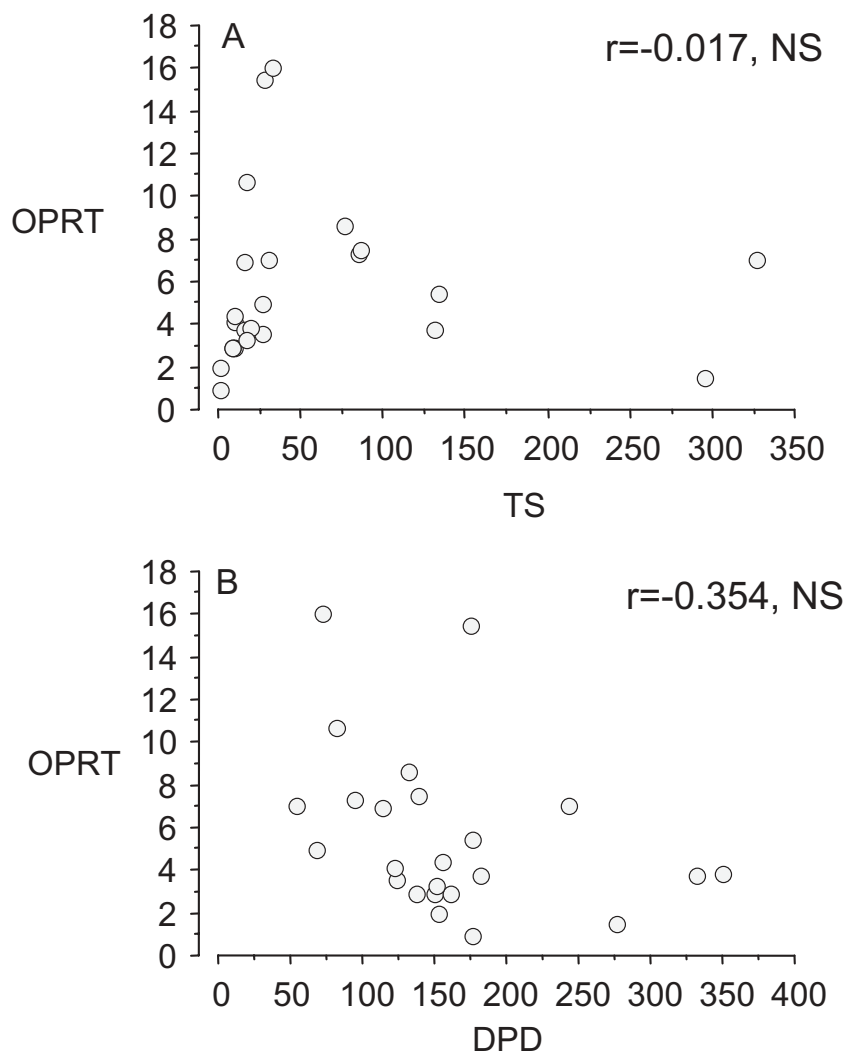

Fig. 4A,B. Correlations between OPRT level and thymidylate synthase (TS) level (A), and between OPRT level and dihydropyrimidine dehydrogenase $(D P D)$ level (B). NS, not significant

Symposium (ASCO-GI). The analysis showed that postoperative adjuvant chemotherapy with S-1 alone significantly improved postoperative survival in patients with stage II/III gastric cancer who had undergone D2 gastrectomy. Although the overall anticancer effect was significant, anticancer effects in individual cases were not uniform, and a certain number of patients showed chemoresistance to S-1-based chemotherapy [18]. Thus, a novel factor that can predict response to S-1-based chemotherapy is needed.

OPRT is a 5-FU-anabolyzing enzyme that plays a primary role in initial phosphorylation in human cancers $[10,19]$. Orotate phosphoribosyltransferase (EC 2.4.2.10) and orotidine-5'-monophosphate decarboxylase (EC 4.1.1.23) are involved in the reactions of adding ribose- 5 ' -phosphate to orotic acid and in the decarboxylation of orotidine-5'-monophosphate, respectively. In vivo, OPRT catalyzes the final two steps in de-novo pyrimidine synthesis [20]. Three distinct pathways by which 5-FU is activated into nucleotide have been identified [19]: (1) direct conversion to FUMP by OPRT in the presence of 5-phosphoribosyl-1-pyrophosphate as a cosubstrate; (2) indirect conversion to FUMP in a sequence of reactions with conversion of 5-FU to 5- 
fluorouridine (FUR) 5-FU, activated in vivo by OPRT and catalyzed by uridine phosphorylase with ribose-1phosphate (Rib-1-P); and (3) indirect conversion to fluorodeoxyuridine monophosphate (FdUMP) by 2'deoxy-5-fluorouridine, catalyzed by thymidine phosphorylase with deoxy-Rib-1-P. Among these pathways, the direct pathway converting 5-FU to FUMP has been shown to be predominant in tumor tissues [19,21], which indicates that OPRT activity is the main rate-limiting step in the phosphorylation of 5-FU. OPRT activity is therefore likely to be associated with the anticancer effects of 5-FU.

The present study demonstrated that OPRT levels were high in well-differentiated-type carcinoma and localized-type carcinoma, and in patients with medullary stroma. Despite the association of OPRT with histological characteristics, however, OPRT levels were unrelated to pathological stage, which is in contrast with the results of a previous report of gastric carcinoma [22]. Mizutani et al. [23] reported that the enzyme activity of OPRT in bladder carcinoma was associated with clinical stage and histological grade. Our results, however, reflect the biological characteristics of gastric carcinoma. Because it has been shown that histological type and the type of local infiltration are associated with prognosis in gastric carcinoma [24, 25], the association of OPRT with clinicopathological characteristics should be accounted for in survival analysis after neoadjuvant or adjuvant chemotherapy.

In the present study, OPRT levels in gastric carcinoma tissue were significantly higher than those in normal gastric mucosa, and this was particularly significant in intestinal-type gastric carcinoma. One possible explanation for this finding is that because OPRT is the enzyme related to pyrimidine synthesis in vivo, higher OPRT levels would be expected in tissues with high cell proliferative activity [26]. In fact, immunohistochemical analysis has shown that OPRT is also expressed in normal gastrointestinal epithelial cells [15]. Previously reported immunohistochemical analysis also showed that the intensity of immunoreactivity of OPRT in cancer cells was stronger than that in their normal counterparts; thus, the results of the present study support the immunohistochemical expression of OPRT in carcinoma tissue and normal gastric mucosa. Therefore, it seems that fluoropyrimidine is phosphorylated mainly in carcinoma tissues, and this indicates that OPRT plays an important role in activating fluoropyrimidine in carcinoma tissue.

In the present study, no significant correlation existed between OPRT levels and levels of TS or DPD in gastric carcinoma tissues. We previously reported no correlation of OPRT levels with thymidine phosphorylase [11]. Because OPRT, TS, and thymidine phosphorylase have been recognized as possible factors that determine sensitivity to DIF, OPRT may be used to determine sensitivity to DIF independently of TS or thymidine phosphorylase.

In summary, although the OPRT level was high in well-differentiated and localized-type carcinomas, OPRT levels were not correlated with the pathological stage of gastric carcinoma. OPRT levels were significantly higher in gastric carcinoma tissue than in normal gastric mucosa, but no correlation existed between OPRT levels in gastric carcinoma tissue and those in normal gastric mucosa. OPRT levels were not correlated with levels of TS or DPD, which suggests that OPRT is a novel factor that is independent of other previously known enzymes related to 5-FU metabolism. Along with our previous observations, suggesting that there is a significant association between the OPRT level and sensitivity to DIF [11], these results suggest that the OPRT level in gastric carcinoma tissues is an independent variable that can predict sensitivity to DIFs. Given the results of the present preclinical study, further clinical study regarding the prognostic significance of OPRT levels in gastric carcinoma tissues in patients with advanced gastric carcinoma undergoing neoadjuvant and/or adjuvant chemotherapy with a DIF is required to confirm the value of OPRT for predicting sensitivity to DIF.

Acknowledgments This work was supported in part by a Grant-in-Aid for Scientific Research (no. 17590321) from the Japan Society for the Promotion of Science. This study was also supported in part by a grant from Fujita Health University.

\section{References}

1. Desai AM, Pareek M, Nightingale PG, Fielding JW. Improving outcomes in gastric cancer over 20 years. Gastric Cancer 2004;7:196-201.

2. Allum WH, Powell DJ, McConkey CC, Fielding JW. Gastric cancer: a 25-year review. Br J Surg 1989;76:535-40.

3. Lordick F, Siewert JR. Recent advances in multimodal treatment for gastric cancer: a review. Gastric Cancer 2005;8:78-85.

4. Maehara Y. S-1 in gastric cancer: a comprehensive review. Gastric Cancer 2003;6 (Suppl 1):2-8.

5. Schoffski P. The modulated oral fluoropyrimidine prodrug S-1, and its use in gastrointestinal cancer and other solid tumors. Anticancer Drugs 2004;15:85-106.

6. Shirasaka T, Nakano K, Takechi T, Satake H, Uchida J, Fujioka A, et al. Antitumor activity of $1 \mathrm{M}$ tegafur-0.4M 5-chloro-2,4dihydroxypyridine-1 $\mathrm{M}$ potassium oxonate (S-1) against human colon carcinoma orthotopically implanted into nude rats. Cancer Res 1996;56:2602-6.

7. Lenz HJ, Lee FC, Haller DG, Singh D, Benson AB, Strumberg $\mathrm{D}$, et al. Extended safety and efficacy data on S-1 plus cisplatin in patients with untreated, advanced gastric carcinoma in a multicenter phase II study. Cancer 2007;109:33-40.

8. Shirasaka T, Shimamato Y, Ohshimo H, Yamaguchi M, Kato T, Yonekura K, et al. Development of a novel form of an oral 5fluorouracil derivative (S-1) directed to the potentiation of the 
tumor selective cytotoxicity of 5-fluorouracil by two biochemical modulators. Anticancer Drugs 1996;7:548-57.

9. Shirasaka T, Nakano K, Takechi T, Satake H, Uchida J, Fujioka A, et al. Antitumor activity of $1 \mathrm{M}$ tegafur- $0.4 \mathrm{M}$ 5-chloro-2,4dihydroxypyridine-1M potassium oxonate (S-1) against human colon carcinoma orthotopically implanted into nude rats. Cancer Res 1996;56:2602-6.

10. Fukushima M, Murakami Y, Suzuki K. The analysis of the innate pathways of 5-fluorouracil phosphorylation in human gastrointestinal cancer cell lines in vitro and in vivo. Oncol Rep 1997;4: 1189-94.

11. Sakurai Y, Sakamoto K, Sugimoto Y, Yoshida I, Masui T, Tonomura S, et al. Orotate phosphoribosyltransferase levels measured by a newly established enzyme-linked immunosorbent assay in gastric carcinoma. Cancer Sci 2006;97:492-8.

12. Sakamoto K, Sugimoto Y, Miyadera K, Oka T, Fukushima M. Preparation of anti-OPRT antibody for immunochemical detection. Jpn J Cancer Chemother 2005;32:653-8.

13. Sakurai Y, Sakamoto K, Sugimoto Y, Yoshida I, Masui T, Tonomura S, et al. Establishment of enzyme-linked immunosorbent assay for quantification of orotate phosphoribosyltransferase measured in gastric carcinoma. Jpn J Cancer Chemother 2005; 32:1017-22.

14. Japanese Gastric Cancer Association. Japanese classification of gastric carcinoma: 2nd English edition. Gastric Cancer 1998;1:10 24.

15. Kamoshida S, Sakamoto N, Matsuoka H, Sakurai Y, Sakamoto K, Sugimoto Y, et al. Heat-assisted stretching of paraffin sections on hot plate weakens immunoreactivity of orotate phosphoribosyltransferase. Acta Histochem Cytochem 2005;38:69-74.

16. Okabe H, Tsujimoto H, Fukushima M. Preparation of the antibodies against recombinant human thymidylate synthase for the detection of its intratumoral levels and the application to sensitivity-study of 5-fluorouracil. Oncol Rep 1997;4:685-90.

17. Kurebayashi J, Yamamoto Y, Udagawa K, Okubo S, Fukushima M, Sonoo H. Establishment of enzyme-linked immunosorbent assays for thymidylate synthase and dihydropyriminide dehydrogenase in cancer tissues. Oncol Rep 2004;11:973-9.

18. Kamoshida S, Suzuki M, Shimomura R, Sakurai Y, Komori Y, Uyama I, et al. Immunostaining of thymidylate synthase and p53 for predicting chemoresistance to S-1/cisplatin in gastric cancer. Br J Cancer 2007;96:277-83.

19. Peters GJ, van Groeningen CJ, Laurensse EJ, Pinedo HM. A comparison of 5-fluorouracil metabolism in human colorectal cancer and colon mucosa. Cancer 1991;68:1903-9.

20. Suttle DP, Bugg BY, Winkler JK, Kanalas JJ. Molecular cloning and nucleotide sequence for the complete coding region of human UMP synthase. Proc Natl Acad Sci U S A 1988;85: 1754-8.

21. Peters GJ, Laurensse E, Leyva A, Lankelma J, Pinedo HM. Sensitivity of human, murine, and rat cells to 5-fluorouracil and 5'deoxy-5-fluorouridine in relation to drug-metabolizing enzymes. Cancer Res 1986;46:20-8.

22. Ochiai T, Sugitani M, Nishimura K, Noguchi H, Okada T, Ouchi $\mathrm{M}$, et al. Impact of orotate phosphoribosyl transferase activity as a predictor of lymph node metastasis in gastric cancer. Oncol Rep 2005;14:987-92.

23. Mizutani Y, Wada H, Fukushima M, Yoshida O, Nakanishi H, $\mathrm{Li}$ YN, et al. Prognostic significance of orotate phosphoribosyltransferase activity in bladder carcinoma. Cancer 2004;100: 723-31.

24. Popiela T, Kulig J, Kolodziejczyk P, Sierzega M. Long-term results of surgery for early gastric cancer. Br J Surg 2002;89:1035-42.

25. Nakamura K, Ueyama T, Yao T, Xuan ZX, Ambe K, Adachi Y, et al. Pathology and prognosis of gastric carcinoma. Findings in 10000 patients who underwent primary gastrectomy. Cancer 1992;70:1030-7.

26. Fujii R, Seshimo A, Kameoka S. Relationships between the expression of thymidylate synthase, dihydropyrimidine dehydrogenase, and orotate phosphoribosyltransferase and cell proliferative activity and 5-fluorouracil sensitivity in colorectal carcinoma. Int J Clin Oncol 2003;8:72-8. 